\title{
RAT BONE MARROW MESENCHYMAL STEM CELLS ISOLATION, CULTIVATION AND DIFFERENTIATION
}

\author{
Emoke PALL*, PhD Student \\ Professor loan GROZA*, PhD \\ Olga SORIŢĂU**, PhD, researcher \\ Ciprian TOMULEASA**, Student, researcher \\ Assistant Mihai CENARIU*, PhD \\ Daria GROZA***, PhD student \\ Teodora VLASIU*, PhD Student
}

\begin{abstract}
Bone marrow stromal cells (MSCs) represent a heterogeneous population derived from the non-blood-forming fraction of bone marrow that regulates hematopoietic cell development. In vitro, adult mesenchymal stem cells resident in this bone marrow fraction differentiate into bone, cartilage, and fat. Because MSCs can be easily obtained using a simple bone marrow aspiration and show extensive capacity for expansion in vitro, these cells have been considered as candidates for cell therapy. The aim of this study was to purify rat MSCs from adult bone marrow and to functionally characterise their abilities to differentiate along diverse lineages. Our data demonstrate that we successfully isolated, culture-expanded and differentiated a relatively homogeneous population of MPCs from adult rat bone marrow.
\end{abstract}

Keywords: mesenchymal stem cells, culture expansion, osteogenic nodules, differentiation, rat bone marrow stem cells

Stem cell research has become an important field of study for molecular, cellular, and clinical biology as well as pharmaco-toxicology $(1,3,4,5,8)$. This cells have a strong proliferative and unlimited self-renewal potential and are multipotent $(4,8)$.

Mesenchymal stem cells (MSCs) represent a population of the bone marrow microenvironment. MSC are non-haematopoietic stromal cells that were first isolated from the bone marrow (BM) but subsequently from other adult connective tissues $(6,7)$ which have been explored as a promising

\footnotetext{
* University of Agricultural Sciences and Veterinary Medicine, Faculty of Veterinary Medicine, Department of Veterinary Reproduction, Obstetrics and Gynecology, 3-5 Manastur Street, 400372 Cluj-Napoca, Romania, tel. +40 264596384 ext. 163 , email: pallemoke@yahoo.com

** "Prof. dr. Ioan Chiricuță" Oncological Institute, Cluj-Napoca

*** "Iuliu Hațieganu“ University of Medicine and Pharmacy, Cluj-Napoca
}

Cluj Veterinary Journal, 15(1)/2009, pp. 29-32 
treatment in tissue regeneration. They exhibit multilineage differentiation capacity being capable to give rise to diverse cells like osteoblasts $(2,9)$, chondrocytes, adipocytes, myocytes, tenocytes and possibly neural cells (7).

We describe a protocol for isolation of mesenchymal stem cells mononuclear cells from adult rat bone marrow.

\section{Materials and Methods}

MSCs were collected from the long bones of three adult Wistar rats (100- 150 g). The animals were killed by cervical dislocation. Their femurs and tibiae were carefully dissected of adherent soft tissue, the epiphyses were removed with a rongeur and bone marrow was flushed out by inserting a syringe needle $(21 \mathrm{G})$ into one end of the bone. Washing bone marrow were used DMEM/F12 (Gibco) medium supplemented with FCS 10\% and antibiotic-antimicotic (100x) (Gibco)1\%.

The aspirant was filtered through a $70 \mathrm{~mm}$ filter (Falcon) to remove bone fragments and then centrifuged at $1000 \mathrm{rpm}$ for $10 \mathrm{~min}$. The cell pellet obtained (containing both haematopoietic cells and marrow stromal cells) was then suspended in MSCs growth medium (DMEM/F12 1x, 10\% fetal calf serum, 1\% antibiotic-antimicotic). The cells were resuspended in normal culture medium to a final concentration of $5 \times 10^{6}$ viable cells per $\mathrm{ml}$ and were then plated on $75 \mathrm{~cm}^{2}$ flasks and left for 72 h. The culture dishes were washed with PBS $+10 \%$ FCS to leave an adherent layer of cells containing marrow stromal cells. Confluent primary cultures were passaged, split, and re-plated; this cycle was repeated three times.

Osteogenic differentiation was induced by culturing MSCs for 2 weeks in DMEM (Gibco) supplimented with 10\%FCS, $10^{-7}$ dexamethasone, $10 \mathrm{mM} \beta$-glicerophosphate, $1 \mu \mathrm{g} / \mathrm{ml}$ insulin, $50 \mu \mathrm{g} /$ $\mathrm{ml}$ ascorbic acid, 10ng/ml BMP2, 2ng/ml TGF $\beta$, with a medium change every 2 days. At the end of the cultivation period, the cells were fixed with $4 \%$ paraformaldehide (Sigma) for $10 \mathrm{~min}$. The cultures were examinated for identification of osteopontin positive cells and colonies according to the manufacturer's instructions. They were then incubated separately with the primary antibodies to anti osteopontin (Sigma), followed by incubation with secondary fluorochromes FITC (anti-goat, diluted 1:100; Sigma). After antibody application, the cells were incubated with a Hoechst nuclear marker solution (diluted $1 \mathrm{mg} / \mathrm{ml}$ in distilled water; Sigma) for $15 \mathrm{~min}$ at $37^{\circ} \mathrm{C}$. Following washing in PBS the preparations were mounted under coverslips, examined at high magnification under a fluorescence microscope (Zeiss Axioplan 2).

\section{Results and Discussion}

For the mesenchymal stem cells recovery we used rats from Wistar line. The bone marrow was recovered by repeated washing of the medullar channel with DMEM/F12 supplemented with FCS.

Rat MSCs obtained from standard purification methods and maintained in adherent culture consist of a morphologically heterogeneous population (Fig. 1A).

After 72 hours from the recovery was made the first medium change, after which was observed the emergence of two major subpopulations (Fig. 1 B,C)); cells with an elongated cells with two processes that extend in opposite directions from the cell body and pronounced bipolarity and other groups of polygonal cells with or without short processes. The first passage was made after 12 days (Fig. 1D) from the recovery when it was identified the emergence of some cellular colonies (Fig. 1C). 

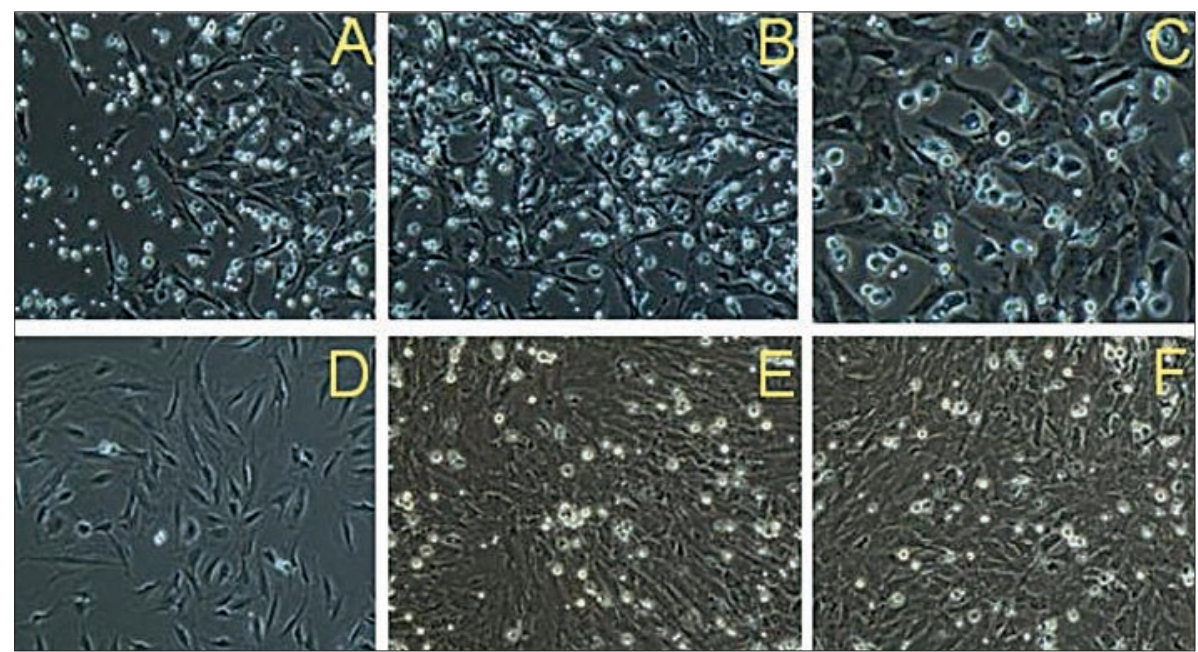

Fig. 1. Photomicrographs of rat mesenchymal stem cells after harvest $24 \mathrm{~h}(\mathrm{~A})$, at 3 days $(B)$, at 4 days $(C)$, small colonies (D), after 1st passage (E), 3nd passage $(F)$

After passage 3 (Fig. 1F) the culture was treated with osteogenic medium, and the medium change was made every other day (Fig. 2A). After 4 days from adding the osteogenic medium we observed the emergence of some nodules (Fig. 2B) of different sizes irregularly dispersed.

Fig. 2. Phase contrast images of rat mesenchymal stem cells osteogenic differentiation; cells with morphology changed (A), and nodules of different sizes (B)

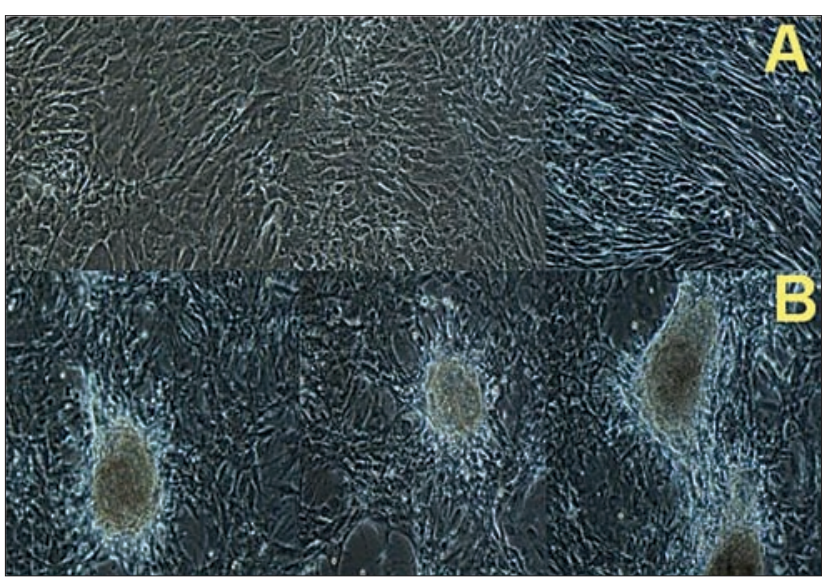

After the examination with the inversed microscope was stated the growth in dimension of the nodules, fact also macroscopically visible. The cells around the nodules also suffered pronounced morphological modifications. Initially the cells presented a fusiform phenotype with some fine prolongations, followed by their transformation in round cells of different sizes. Towards the end of the experiment we observed a cellular juxtaposition: the fist layer of elongated cells over which it was stated the superposition of a layer of round cells.

The osteogenic differentiation was identified with the aid of the osteopontin antibody. At the fluorescence microscope we identified the fact that the nodules formed were osteopontin positive that demonstrates that these nodules were formed from osteoblasts.

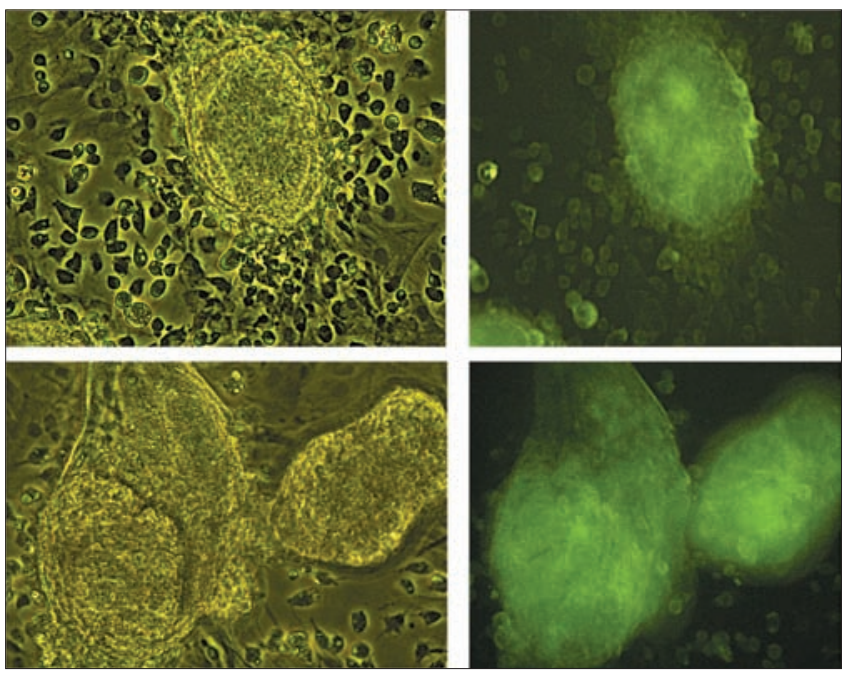

Fig. 3.- Immunohistochemical highlighting of osteopontin positive cells and nodules 


\section{Concusion}

In conclusions MSCs from rat bone marrow are relatively easily isolated from the bone marrow and can be manipulated in vitro, they could be good candidates for the development of various therapeutic modalities aimed to regenerate mesenchymal tissues. Better understanding of the molecular mechanism directing the differentiation of BMCs will eventually allow us to properly manipulate BMCs both in vivo and ex vivo for regeneration of complex tissues and organs. Less passaged BMCs cultured with osteoinductor medium proved to possess in vivo osteogenic potential. This can be applicable to use cell therapy in the repair of bone defects. In clinical feasibility, however, large cell numbers with osteogenic potential will be required. Further investigations are needed to establish the culture conditions that permit the rapid expansion of mesenchymal stem cells while retaining their potential for differentiation.

\section{References}

1. Bianco P., Riminucci M., Gronthos S., Robey P.G., 2001, Bone marrow stromal stem cells: nature, biology and potential applications, Stem Cells 19/180-192;

2. Fumiaki Sugiura, Hiroshi Kitoh, Naoki Ishiguro, 2004, Osteogenic potential of rat mesenchymal stem cells after several passages, Biochemical and Biophysical Research Communications 316, 233-239;

3. Groza I.S., Emoke Pall, Olga Soriţău, D.D. Ciupercescu, Laura Cătană, 2007, Isolation, characterization and differentiation pf stem cells from bone marrow of mice CD1 strain, Revista Română de Medicină Veterinară, vol.17, 4, pag.71-84;

4. Guanbin Song, Yang Ju, Hitoshi Soyama, 2008, Growth and proliferation of bone marrow mesenchymal stem cells affected by type I collagen, fibronectin and BFGF, Materials Science and Engineering C MSC-02266; no. of pages 5;

5. Jose J.M., Alejandro E., Paulette Conget, 2001, Mesenchymal stem cells, Minireview, Society for Experimental Biology and Medicine;

6. Pountos I, Giannoudis PV., 2005, Biology of mesenchymal stem cells. Injury; 36:S8-S12.

7. Pountos I, Jones E, Tzioupis C, 2006, Growing bone and cartilage. The role of mesenchymal stem cells. J Bone Joint Surg Br.; 88:421-6;

8. Tarja A.J., William S., Xuan Y., Michael I.C., Chi V.D., saul J.S., 2006, Isolation of bone marrow derived stem cells using density gradient separation, Experimental Mematology, 35/335-341;

9. Xiaoli W., Hiroko H., Shigeru T., Yasushi A., Qiang L., Wenhao C., Jianfeng W., Changye WS., Tomomi M., Satoshi O., Quing L., Tianxue F., Hongxue F., Zhexiong L., Gershwin M.E., Sususmu I., 2005, Characterization of mesenchymal stem cells isolated from mouse fetal bone marrow, Stem Cells, 24/482493; 